\title{
Can Knowledge Management be Open Source?
}

\author{
Charmaine C Pfaff ${ }^{1}$ and Helen Hasan ${ }^{2}$ \\ 1 University of Wollongong, Faculty of Commerce, Information Systems, \\ Wollongong 2522, Australia \\ hasan@uow.edu.au \\ WWW home page: http://www.uow.edu.au/commerce/infosys/hasanh.html \\ 2 University of Wollongong, Faculty of Commerce, Information Systems, \\ Wollongong 2522, Australia \\ ccp02@uow.edu.au
}

\begin{abstract}
As we move further into a knowledge economy where collaboration and innovation are increasingly central to organisational effectiveness, enterprises need to pay more attention to the informal networks that exist within the organisation. Wikis may provide a more appropriate knowledge management capability and environment to capture tacit knowledge. Where traditional organisational cultures see that knowledge management must be tightly protected, Wikis opt for an open source approach where knowledge is shared and distributed for innovation to continue. This paper aims to explicate more participatory organisational processes of creation, accumulation and maintenance of knowledge. It uses Activity Theory as a framework to describe the components of an activity system where a Wiki is a tool mediating employee-based knowledge management activities and thereby democratising organisational knowledge.
\end{abstract}

\section{Introduction}

As organisations aim at moving knowledge from the realm of the individual into the hands of the organisation, they often resort to expensive Knowledge Management Systems (KMS) with data mining and search engines to organize and access large volumes of documents. Although traditional business logic dictates that there must be organisational controls to ensure conformity so that tasks can be defined and measured, they stifle creativity and initiative, constraining the design of the next generation KMS. In practice, the new business environment requires a KMS that performs better on fewer rules, some specific information and greater freedom. The goals of this paper are to analyse the essential elements of organisational

Please wse the following format when citing this chapter.

Pfaff, C.C. and Hasan, H., 2007, in IFIP International Federation for Information Processing, Volume 234, Open Source Development, Adoption and Innovation, eds. J. Feller, Fitzgerald, B., Scacchi, W., Sillitti, A., (Boston: Springer), pp. $59-70$. 
knowledge, KM and knowledge workers in creating a more cooperative and democratic KM and the potential of using corporate Wikis as new generation KMS.

\section{Managing Knowledge and Knowledge Workers}

\subsection{The nature of knowledge}

The current corporate interest in knowledge is based on a realisation that emerging economic imperatives, coupled with social and industrial restructuring, demand a more rigorous approach to the exploitation of knowledge as an organisational resource. Organisational knowledge can be about what employees understand about historical knowledge inherent in the organisation such as the knowledge about customers, products, processes, errors, and successes. Various streams of KM research have emerged. However, the differences in interpretation and definition have become a matter of contention.

It is challenging to scan the human mind for tacit knowledge (knowledge that is embedded in a person's mind and cannot be expressed easily and explicitly) because most individuals may know more than they think they know. The sense meaning making capacity of the human mind may evoke tacit knowledge as a response to new and unfamiliar stimuli or situations that may not fit previously recognised scenarios. In addition, it ignores the possibility that additional knowledge resides in the relationships between employees and in the legacy of previous employees embedded in organisational memory and culture.

From an IS perspective, knowledge is the top of the data-information-knowledge hierarchy where information is meaningful, processed data and knowledge is actionable information, separating knowledge from information or data (Handzic \& Hasan 2003). This view of knowledge reinforces the value of using CHAT for research on KM because it can extract actionable meaning from unstructured or illstructured information, social interaction patterns, and deep rooted motives of knowledge workers.

\subsection{Problems with managing knowledge}

Many KMS have not met their original business objectives because there is an assumption that all relevant knowledge, including tacit knowledge should be extracted from knowledge workers and stored through well-established institutional processes in well-designed knowledge repositories (AS5037[Int] 2003). The process of building these repositories has been criticised as being time-consuming, laborious, and costly. The process of building these repositories has been criticised as being time-consuming, laborious, and costly. Viewed by many as a superficial implement of management, they are often not kept up-to-date and are rarely accessed when real knowledge is sought (Klint \& Verhoef 2002).

Hart \& Warne (2005) have stressed that it is detrimental to manage organisational knowledge because knowledge by its very nature cannot be managed in the traditional sense. KM cannot be fostered in settings where people feel 
pressured as it makes them less motivated to engage in dialogue. Often, employees hoard their knowledge because their contributions do not benefit their careers and becomes an additional burden to their already heavy workloads (Lam \& Chua 2005). Hence, the authors support the Australian Standard (AS 5037-2005) definition of $\mathrm{KM}$ : "KM is concerned with innovation and sharing behaviours, managing complexity and ambiguity through knowledge networks and connections, exploring smart processes, and deploying people-centric technologies."

The focus on work practices reveals how community members conceptualise the work they perform and the synergistic roles of the community and its members in the processes of knowledge production. 'Knowledge work' is not restricted to the work practices of individuals and teams that create and exploit knowledge (Burstein \& Linger 2003), knowing 'how' and 'who' you know are as important as what you know. Understanding how knowledge workers work and their needs will help them become more productive.

\section{The Wiki Way}

\subsection{Open source revolution}

The notion of creative collaborative work is not new. Its best known propagator is the open source software development. The open source movement (OSM) began as experiments in software democracy that crossed institutional and geographical boundaries. It has achieved a momentum in motivating people to work together in self organised groups on common projects and making them available on the Internet for use or modification. The OSM is fulfilling the original promise of the Internet and promoting the Internet culture where people can work together in an environment that supports access to information. Already, it has inspired the emergence of an ecosystem of other projects such as Creative Commons sharing media resources and Wikipedia. KMS can learn some lessons from its success such as simplicity in design, frequent reviewing and testing, a skilled and devoted group of volunteers and developers, and simple but effective rules to govern the community (Wagner 2006). It is our contention that new ICT tools such as the corporate Wiki can be the enabler to effect changes for the better in organisations. For example, organisations that adopt a rigorous 'best practices' approach find it extremely challenging not to be caught in the death spiral (Nadler and Shaw 1995) of doing more of the same better and better with diminishing marginal returns (Drucker 1994). The corporate Wiki provides an environment to ensure that such practices remain open to critique, adaptation, and replacement.

\subsection{Conversational Technology}

New conversational technologies such as email, discussion forums, chatrooms, Weblogs and Wikis are now connecting and supporting liberated knowledge exchanges much as transportation systems and cities on the ground have always done. Interconnected networked structures of social interaction and creative activity are emerging as a part of the civil digital 
culture and, less rapidly, in the knowledge work of organisations. Conversational technologies are seen as tools to support work units and the individual knowledge worker. It is the corporate Wiki that is of most interest to the field of KM because it can be developed by end users through collaboration (Hasan \& Pfaff 2006a, Wagner 2006).

A Wiki is a web-based application that allows many participants to write collaboratively, where anyone can start a new page or edit an existing one. Such documents can be supported by the web with hyperlinks to anywhere on the World Wide Web including text, image and video. 'Wiki Wiki' in Hawaiian means 'quick' or 'fast' which refers to the quick editing processes (Leuf \& Cunningham 2005). A Wiki is a collection of interlinked HTML web pages. Changes are logged and viewed online instantly and can be reverted to its original state. A Wiki can be accessed from any web browser and no other special tools are needed to create and edit existing pages. A Wiki is an evolving knowledge repository where users are encouraged to make additions to this repository by adding new documents or working on existing ones (Pfaff \& Hasan 2006). The most well known example of a Wiki is Wikipedia ${ }^{1}$, an online encyclopaedia exemplifying the open source ideal.

\subsection{Factors contributing to the rise of Wikipedia}

The openness of Wikipedia as a publicly editable website is a phenomenal motivating factor for people to work together and share their knowledge to teach the world. Emigh and Herring (2005) compared traditional printed sources with Wikipedia articles and found them stylistically indistinguishable and citing Wikipedia articles in news and other media have become common (Lih 2004). Wiki users feel a sense of ownership when they see their work online and want to "collaborate radically", a feature of the OSM where anyone can edit another person's work. Collaboration avoids bottleneck complications if there is an individual author and the constant editing refines the article (Sanger 2005). The neutral policy of a Wiki allows everyone to air their views while at the same time respecting divergent views.

Nupedia is the predecessor to Wikipedia which began in 2000. Its founders wanted volunteers to write, edit and review articles as they would for a printed, for profit published encyclopaedia. Nupedia was terminated in 2003 because of its server problems, intolerance to writers who are not experts and mainly, its complicated review process. (Rosenzweig 2006, Sanger 2005). The founders of Wikipedia reduced the turnaround time to edit and review Wikipedia articles, to overcome the participatory problem.

\subsection{Wikipedia criticisms}

The main allegation about Wikipedia is that the information varies in quality. To investigate this claim, Rosenzweig (2006) compared 25 Wikipedia biographies against comparable entries in Encarta and American National Biography Online. Although both publications have multimillion-dollar budgets, it was found that

${ }^{1}$ http://en.Wikipedia.org 
Wikipedia articles were favourably written. Wikipedia is also accurate in reporting names, dates, and events in U.S. history. Wikipedia surpasses Encarta but not American National Biography Online in coverage and matches Encarta in accuracy. In another study, a German computing magazine engaged experts to compare Wikipedia articles in 22 different fields in the three leading German-language digital encyclopaedias. It rated Wikipedia first with a 3.6 on a 5-point scale, Brockhaus Premium scored 3.3 and Encarta 3.1. (Kurzidim 2004). A British scientific magazine, Nature, asked academic scientists to do a blind review of 42 science entries in Wikipedia and Encyclopaedia Britannica. Wikipedia contained around four inaccuracies and Britannica, had three (Giles 2005).

\subsection{Adapting the Wiki in a corporate setting}

Looking back at other technologies e.g. personal computers, email and instant messaging that enjoyed widespread popularity; management needs to think of how to adapt already popular social tools for corporate use because the impact of grassroots marketing should not be overlooked. Nevertheless, some of the problems facing Wikipedia are reflected in corporate Wikis. (Hasan \& Pfaff 2006b, Wei et. al 2005). The principal dilemma of a Wiki is that, while its anarchic nature is desirable for fostering open debate without censorship, it raises questions whether the information is authoritative and credible, thus inhibiting its usefulness. Yet a critical factor to bear in mind is that Wikipedia is a public online Wiki. Employees who make contributions to the corporate Wiki are employed by the organisation as specialists whose opinions will be highly regarded by their organisations as trusted and authoritative.

The informal network approach that is currently favoured in a Wiki, may make some companies believe that their data quality will be affected and that system errors will occur. Their centralised and highly structured environment will make it difficult to adopt a 'community approach' towards knowledge acquisition. The problems of irresponsible behaviour and accountability issues for fraudulent data can be avoided because employees using a corporate Wiki will not be using "handles" but their real names to login to edit the Wiki. This means that every post or edit could be attributed to an individual employee. A footnote can be included to remind employees that usage could be traced back to them to deter intentional misuse. Wikis have a rollback feature which could be used by administrators to repair deletions or misuse. Daily backups can preserve the Wiki database against loss of data in case of system failures (Auger et. al. 2004).

Employees should not see the corporate Wiki as merely an online shared space and owned by a gatekeeper. The corporate Wiki should instead be seen as an open community process that encourages multiple iterations in the creation of a knowledge repository (Wei et al 2005). 


\section{Cultural Historical Activity Theory (CHAT)}

\subsection{Theoretical basis}

Although CHAT was proposed long before the advent of computers and the Internet, a growing band of researchers recognise that CHAT provides a rich holistic understanding of how people collaborate with the assistance of sophisticated tools in the complex dynamic environments of modern organisations (Thomas \& Torstein 2005, Waycott et. al 2005, Hasan 1999).

The notion of activity is interpreted from the theory of Leontiev (1981) which is based on Vygotsky's psychology. Vygotsky (1978) proposed that all human activity is purposeful, carried out through the use of 'tools' and socially mediated. Tools can manipulate and transform objects but also restrict what can be done within the limitation of the tool, which, in turn, often stimulates improvements to the tool (Verenikina \& Gould 1998), especially in the context of analysing the dialectic interactions between people and technologies, and how they are shaped by human activity.

\subsection{Assumptions of the Activity System}

The analysis begins with the identification and explication of the central activity and then looks at those activities that are linked to it (Hasan 2003a). As described in Hasan (2003b), Engeström (1987), Kuutti and Virkunnen (1995), an activity system normally has one central activity, which is the focal point of holistic investigation, surrounded by other activities with some link to the central activity.

Figure 1 shows the activity as the engagement of a subject toward a certain goal or objective where the project team is a collective subject composed of individuals who bring different skills and understandings to bear on a common object, the corporate Wiki. The purpose of the Wiki activity is to create, share and manage knowledge in the form of an encyclopaedia, which will persist over time while the participants may change. The core activity (object), for which a corporate Wiki is used, is not KM per se but knowledge work. There is a dialectic relationship between knowledge and work, expressed by the continuous cycle of co-creating work-related knowledge in a form that is meaningful for knowledge workers to access as needed, through which learning occurs, resulting in more knowledgeable doing and so on.

The tools are the Wiki technology together with social and learning processes within the organisation. Each participant subject brings different personal characteristics that may change over time, including individual motivations, goals, and self perceptions affecting the transformation of goals. Contributions can come from users' personal knowledge, which is related to fields where they feel comfortable and competent such as work projects or knowledge specialisations. 


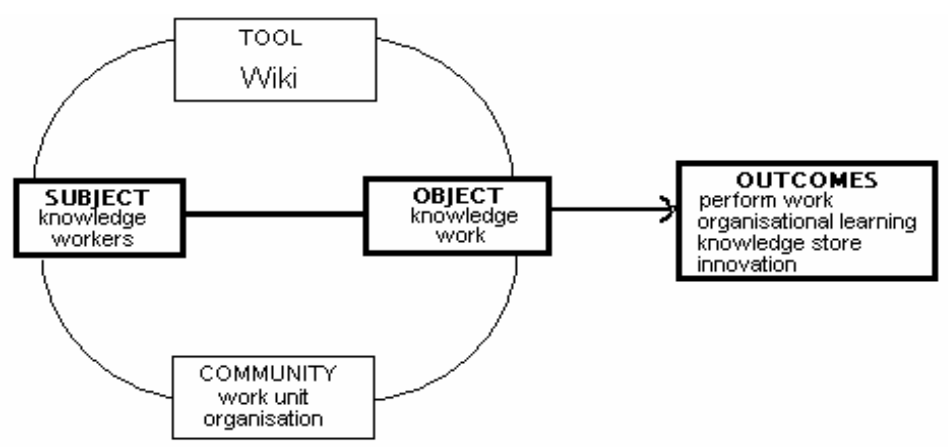

Fig. 1. The core activity of knowledge work mediated by a corporate Wiki

In trying to address the limitations of prior KMS, a corporate Wiki overcomes the barrier of KMS created from the static accumulation of dynamic knowledge. The activities of the knowledge worker are mediated not only by the functions of the corporate Wiki itself, but also by the attitudes and customs of the organisations in giving workers the resources and authority to do so. As knowledge workers operate at the grass roots level, they are in the best position to act as sense makers in a rapidly changing dynamic environment. Knowledge workers can participate as writers and peer reviewers, giving them opportunities to define problems and generate their own solutions, evaluate and revise their solution-generating processes.

Managers can embrace change by building up individuals, and reducing the dependence on strengthening institutions, which in turn has led to the creation of a highly protective culture of the status quo. 


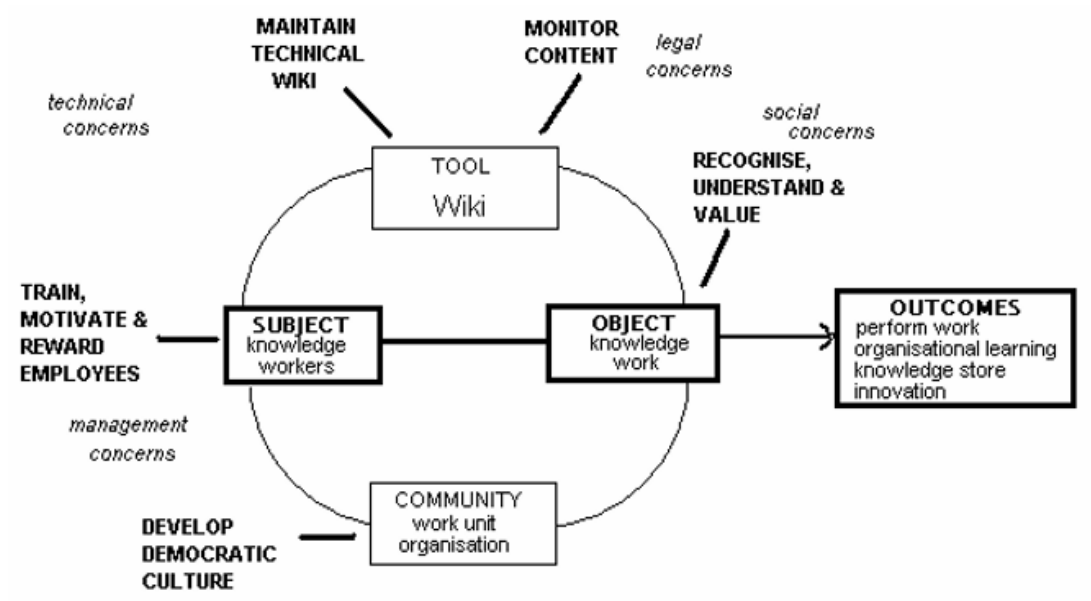

Fig. 2. Elements of secondary activities related to knowledge work using a corporate Wiki

The distinctive attributes of corporate Wikis give us an indication of the technical, legal, social and management concerns that links to the core knowledge work activity shown in Figure 2. Technical concerns include ensuring that there is technical support to install the corporate Wiki and maintain overall quality. The legal aspects such as copyright issues and legal liability will increase the social responsibility of Wiki users as time progresses. The very nature of a Wiki is democratic as it values participation over power. Knowledge workers can only thrive in a culture that respects the minds of knowledge workers and promotes experimentation and rethinking. Democracy raises public awareness of issues such as openness, freedom of information and public accountability (Benkler 2006). A corporate Wiki will redefine the social constructs of the organisation because it places knowledge workers on the same level as management. The main hurdles for management are issues of trust, credibility and authority. Management has been concerned that previous KMS failed to motivate employees to contribute and use its contents (Hasan 2003a). As demonstrated by the popularity of Wikipedia, the corporate Wiki can overcome this participatory problem. However, this may depend on the organisation having a knowledge sharing culture and appropriate job descriptions and incentives in place.

\section{Future Directions}

The revised Australian KM Standard (AS 5037-2005) recognises that an emergent $\mathrm{KM}$ generation requires radical changes to traditional organisational cultures to create environments where knowledge workers are authorised, empowered and encouraged to cooperatively manage their own work practices and knowledge.

From the onset, the organisation needs to appoint a core team of knowledge workers or "Wiki evangelists", who are good writers and experts in their specialised 
fields who can come to a consensus on what the encyclopaedia should look like and "seed" the corporate Wiki. Wiki scribes can help those who are not comfortable with technology or are not fluent writers. The adoption of an incremental principle points out to the non existence of pages which tempts users to create new pages of content e.g. produce an annual report or submit ideas for a group project, as part of the workload. As employees grow more confident, the corporate Wiki can harvest contributions about declarative knowledge (know-what) e.g. 'best practices', business procedures and rules; procedural knowledge (know how) e.g. stories, conversations and other context-rich knowledge, and conceptual knowledge (know why) e.g. principles and laws (Agarwal et al. 1997). If this is made easier using the corporate Wiki than without it, employees may take on board the benefits and readily move to other tasks.

A democratic culture of knowledge sharing reinforces the notion that knowledge workers' reputations are enhanced by participation in collaborative projects, acquire marketable job skills and knowledge; and increasing social recognition and prestige, just as people are rewarded for collaborative professional work. Traditionally, very few powerful people dominate the channels of information. The creation of the Internet has had a democratising effect on the availability and use of information. Things that seem to matter in the real world, such as age, social status, and level of education, are often dismissed as unimportant online. The same democratising effect will be true with Wikis. As it is not easy to transfer the cumulative experience and skills of employees to the organisation, a corporate Wiki can address this problem by being a 'peer production information commons' (Benkler, 2006). A corporate Wiki that is based on an open source model promoting a participatory and bottom up approach, can be common spaces where people share experiences and have unanticipated, un-chosen exposures to the ideas of other people.

Successful collaborations from the OSM such as Linux were created outside the business environment and have become mainstream. Wikipedia's popularity is due to its open, free and collaborative nature that helps meet the challenges of a connected world. If corporate Wikis can borrow elements that contribute to Linux and Wikipedia's success, while at the same time addressing their limitations, corporate Wikis will get an opportunity to prove how mainstream this new generation KMS can become.

\section{References}

Agarwal, R., Krudys, G. and Tanniru, M. 1997, Infusing Learning into the IS Organization. European Journal of Information Systems, 6(1):25-40.

AS5037 [Int] 2003, Interim Australian Standard Knowledge Management. Standards Australia

AS5037 2005, Australian Standard Knowledge Management. Standards Australia.

Benkler, Y. 2006, The Wealth of Networks. (February 20, 2007) http://www.benkler.org/wealth_of_networks/ 
Burstein, F. and Linger, H. 2003, Supporting post-Fordist work practices: A KM framework for dynamic intelligent decision support, Journal of IT\&P special issue on KM, 16(3): 289-305.

Drucker, P. 1994, The Theory of Business. Harvard Business Review, SeptemberOctober, pp. 95-104.

Emigh, W. and Herring, S. 2005, Collaborative authoring on the web: A genre analysis of online encyclopedias. Proceedings of the Hawaii International Conference on System Sciences.

Engeström Y. 1987, Learning by expanding: An activity-theoretical approach to developmental research. Helsinki: Orienta-Konsultit.

Giles, J. 2005, Internet encyclopaedias go head to head, Nature, Dec. 15, http://www.nature.com/nature/journal/v438/n7070/full/438900a.html.

Hart D. and Warne L. 2005, Comparing cultural and political perspectives of data, information and knowledge sharing in organizations, Journal of Knowledge Management

Handzic, M. and Hasan, H. 2003, The search for an integrated KM framework. In Hasan, H. \& Handzic, M. (Eds). Australian Studies in Knowledge Management, UOW Press, pp. 3-34.

Hasan, H. 1999, Integrating IS and HCI using AT as a Philosophical and Theoretical Basis, Australian Journal of Information Systems, 6(2):44-55.

Hasan, H. 2003a, An Activity-based Model of Collective Knowledge. In Proceedings of the 36th Hawaii International Conference on System Sciences, Big Island, Hawaii, USA.

Hasan, H. 2003b, Communities as Activity Systems and other such Frameworks. In Hasan, H., Verenikina, I. and Gould, E.(Eds) Information Systems and AT, Expanding the Horizon, UOW Press, 3, pp.74-95.

Hasan, H. and Pfaff C.C. 2006, The Wiki: a tool to support the activities of the knowledge worker. In Proceedings at the Transformational Tools for $21 \mathrm{st}$ Century (TT21) 2006 Conference. Central Queensland University, Rockhampton, Queensland.

Hasan, H. and Pfaff C.C. 2006, Emergent Conversational Technologies that are Democratising Information Systems in Organisations: the case of the corporate 
Wiki. Proceedings at the ISF: Theory, Representation and Reality conference, ANU, Canberra.

Klint, P. and Verhoef, C. 2002, Enabling the creation of knowledge about software assets. Data and Knowledge Engineering, 41:2-3, 141-158.

Kurzidim, M. 2004, Wissenswettstreit. Die kostenlose Wikipedia tritt gegen die Marktführer Encarta und Brockhaus an. (Knowledge competition: Free Wikipedia goes head to head with market leaders Encarta and Brockhaus). Oct. 4, pp.13239.

Lam, W. and Chua, A. 2005, Knowledge management project abandonment: an exploratory examination of root causes, Communications of the Association of Information Systems, 16: 723-743.

Leontiev, A.N. 1981, Problems of the Development of Mind. Moscow, Progress.

Leuf, B. and Cunningham, W. 2001, The Wiki Way, Quick Collaboration of the Web. Addison-Wesley.

Lih, A. 2004, Wikipedia as Participatory journalism: reliable sources? Metrics for evaluating collaborative media as a news resource. Proceedings of the Fifth International Symposium on Online Journalism, April 16-17, Austin, Texas.

Nadler, D.A. and Shaw, R.B. 1995, Change leadership: core competency for the $21^{\text {st }}$ Century. In Nadler, D.A., Shaw, R.B. and Walton, A.E. (Eds.), Discontinuous Change: Leading Organizational Transformation. San Franscisco, CA: JosseyBass.

Nonaka, I. 1991, The knowledge-creating company. Harvard Business Review, Nov/Dec, pp.96-104.

Pfaff, C.C. and Hasan, H. 2006, Overcoming organisational resistance to using Wiki technology for Knowledge Management. In Proceedings of the $10^{\text {th }}$ Pacific Asia Conference on Information Systems, Kuala Lumpur, Malaysia.

Rosenzweig, R. 2006, Can history be Open Source? Wikipedia and the future of the past. The Journal of American History, 93(1):117-46.

Sanger, L. 2005, The early history of Nupedia and Wikipedia: a memoir. In DiBona, C., Stone, M. and Cooper, D., editors, Open Sources 2.0, O'Reilly Press: Sebastopol, CA. 
Thomas, H. and Torsten, P. 2005, Supporting knowledge work with Knowledge Stance-Oriented Integrative Portals, Proceedings of the European Conference on IS, Regensburg, Germany.

Wagner, C. 2006, Breaking the knowledge acquisition bottleneck through conversational knowledge management. Information Resources Management Journal, 19(1):70-83.

Waycott, J., Jones, A. and Scanlon, E. 2005, PDAs as lifelong learning tools: An AT based Analysis. Learning, Media and Technology, 30(2):107-130.

Wei, C., Maust, B., Barrick, J., Cuddihy, E. and Spyridakis, J. H. 2005, Wikis for supporting Distributed Collaborative Writing. In Proceedings at the Society for Technical Communication, Seattle, pp. 204-209. 